18,12

\title{
Изменение теплопроводности многослойного массива углеродных нанотрубок при его поперечном сжатии
}

\author{
(C) A.B. Савин \\ ${ }^{1}$ Федеральный исследовательский центр химической физики им. Н.Н. Семенова РАН, \\ Москва, Россия \\ ${ }^{2}$ Российский экономический университет им. Г.В. Плеханова, \\ Москва, Россия \\ E-mail: asavin@center.chph.ras.ru
}

Поступила в Редакцию 2 ноября 2021 г.

В окончательной редакции 14 ноября 2021 г.

Принята к публикации 18 ноября 2021 г.

Проведено численное моделирование теплопроводности поперек многослойного массива одностенных углеродных нанотрубок. Изучено влияние на теплопроводность поперечного сжатия массива. Показано, что сжатие массива может происходить однородно, когда все нанотрубки массива одинаково сжимаются, и неоднородно, когда часть нанотрубок сжимается сильно, а другая часть - слабо. При однородном сжатии теплопроводность массива увеличивается, а при неоднородном не меняется и даже, при большом числе слоев, может уменьшатся. Этот эффект особенно ярко проявляется для массивов нанотрубок небольшого диаметра $(D<2 \mathrm{~nm})$.

Ключевые слова: углеродная нанотрубка, массивы нанотрубок, поперечное сжатие, теплопроводность.

DOI: $10.21883 /$ FTT.2022.03.52100.231

\section{1. Введение}

Углеродные нанотрубки (УНТ) имеют форму свернутого в цилиндр листа графена диаметра от $0.4 \mathrm{~nm}$ и длиной до нескольких $\mathrm{cm}$. Впервые топологически похожие молекулярные структуры были получены при термическом разложении окиси углерода на железном контакте [1]. Сами УНТ как идеальные цилиндрические макромолекулы были получены значительно позже как побочные продукты синтеза фуллерена $\mathrm{C}_{60}$ [2]. Интерес к УНТ связан с их уникальными свойствами [3,4]. К настоящему времени нанотрубки с требуемыми геометрическими свойствами (т.е. с нужным диаметром, длиной и хиральностью) могут быть легко синтезированы $[5,6]$ и использованы для получения пучков параллельных УНТ [7,8]. Такие материалы, также называемые лесами или массивами УНТ, обладают еще более превосходными механическими свойствами по сравнению с изолированными нанотрубками из-за ван-дер-Ваальсовых взаимодействий между ними [9].

Изолированные УНТ обладают высокой теплопроводностью [10-14], их теплопроводность может достигать значений выше $3000 \mathrm{~W} / \mathrm{mK}$. Численное моделирование теплопереноса вдоль идеальной одностенной нанотрубки показывает, что коэффициент теплопроводности монотонно растет при увеличении ее длины [15-17]. Детальное моделирование теплопереноса вдоль нанотрубки с индексом хиральности $(6,6)$ показало [18], что теплопроводность идеальной нанотрубки растет прямо пропорционально логарифму ее длины. Макроскопические сборки нанотрубок, такие как их массивы, волокна (пучки) и пленки, обладают более низкой теплопроводностью из-за плохого выравнивания нанотрубок и низкой плотности их упаковок [19-22]. Пленки высоко выровненных УНТ получены в [23], их теплопроводность может достигать значений до $700 \mathrm{~W} / \mathrm{mK}$. В пучке высоко выровненных (параллельных) нанотрубок теплопроводность поперек существенно ниже теплопроводности вдоль пучка. Согласно расчетам [24] поперечная теплопроводность пучка параллельных УНТ $(10,10)$ имеет очень низкое значение $0.05 \mathrm{~W} / \mathrm{mK}$, так как за теплоперенос поперек пучка отвечают слабые ван-дерВаальсовы, а за теплоперенос вдоль - сильные валентные связи. Поперечную теплопроводность можно увеличить, если ввести между соседними нанотрубками межмолекулярные валентные мостики [25,26]. Отметим, что детальное численное моделирование теплопереноса поперек многослойных массивов УНТ не проводилось (рассматривались только отдельные элементы таких массивов).

Нанотрубки обладают высокой продольной (осевой) и относительно слабой поперечной (радиальной) жесткостью. В силу этого при достаточно большом диаметре нанотрубка из-за невалентного взаимодействия атомов ее поверхности может переходить из пустотелой цилиндрической формы в схлопнутое (коллапсированное) состояние [27-33]. Коллапсирование нанотрубок должно существенно влиять на поперечную жесткость и теплопроводность многослойных массивов параллельных УНТ.

Из-за пустотелости УНТ их кристаллы, в отличие от плотных материалов, могут демонстрировать очень 
высокую поперечную сжимаемость в упругой области. Это позволяет использовать массивы УНТ для защиты от ударов и вибраций $[34,35]$. В настоящей работе будет изучено влияние поперечного сжатия многослойного массива одностенных нанотрубок на его теплопроводность. Будет показано, что сжатие массива может приводить не только к повышению теплопереноса поперек массива, но и, в зависимости от степени сжатия, к его понижению. Для моделирования теплопереноса будет использована цепная модель, предложенная ранее для моделирования динамики листов графена [36] и модифицированная далее для анализа структуры массива УНТ [37]. Будет проведено моделирование теплопереноса поперек многослойного массива УНТ при разной степени его сжатия.

\section{2. Цепная модель наноленты и нанотрубки графена}

Рассмотрим массив параллельных одностенных углеродных нанотрубок, лежащий между плоскими многослойными листами графена - см. рис. 1. При поперечном сжатии форма данной молекулярной структуры полностью определяется формой ее поперечного сечения. Пусть нанотрубки массива лежат вдоль оси $z$, а ограничивающие массив листы графена - параллельно плоскости $x z$. В поперечном сечении (в плоскости $x y$ ) массив УНТ будет иметь форму многослойной системы циклических цепочек, лежащих между линейными цепочками. Поэтому массив удобно описать с помощью двухмерной модели, в которой листам графена соответствуют линейные [36], а нанотрубкам - циклические молекулярные цепочки [38].

При поперечном сжатии форма сечения нанотрубки полностью описывает ее деформацию. Поэтому сжатие пучка параллельных нанотрубок может быть описано как деформация их поперечных сечений. Такой подход позволяет значительно снизить размерность моделируемой молекулярной системы. Данная модель была ранее успешно использована для моделирования рулонов нанолент графена [36], накручивания нанолент на нанотрубки [38], поперечного сжатия пучка нанотрубок [37] и для анализа механических свойств одностенных и многостенных нанотрубок, расположенных на плоских подложках $[39,40]$.

При сечении листа (наноленты) графена в направлении зиг-заг он имеет вид однородной цепочки с шагом $R_{0}=r_{c} \sqrt{3} / 2=1.228 \AA\left(r_{c}=1.418 \AA-\right.$ длина валентной связи $\mathrm{C}-\mathrm{C})$. Гамильтониан поперечного сечения листа графена будет иметь вид

$$
H_{r}=\sum_{n=1}^{N_{r}}\left[\frac{1}{2} M\left(\dot{\mathbf{u}}_{n}, \dot{\mathbf{u}}_{n}\right)+V\left(R_{n}\right)+U\left(\theta_{n}\right)+W_{0}\left(y_{n}\right)\right],
$$

где двухмерный вектор $\mathbf{u}_{n}=\left(x_{n}, y_{n}\right)$ задает координаты $n$-го атома цепи, $M=12 m_{p}$ - масса атома углерода

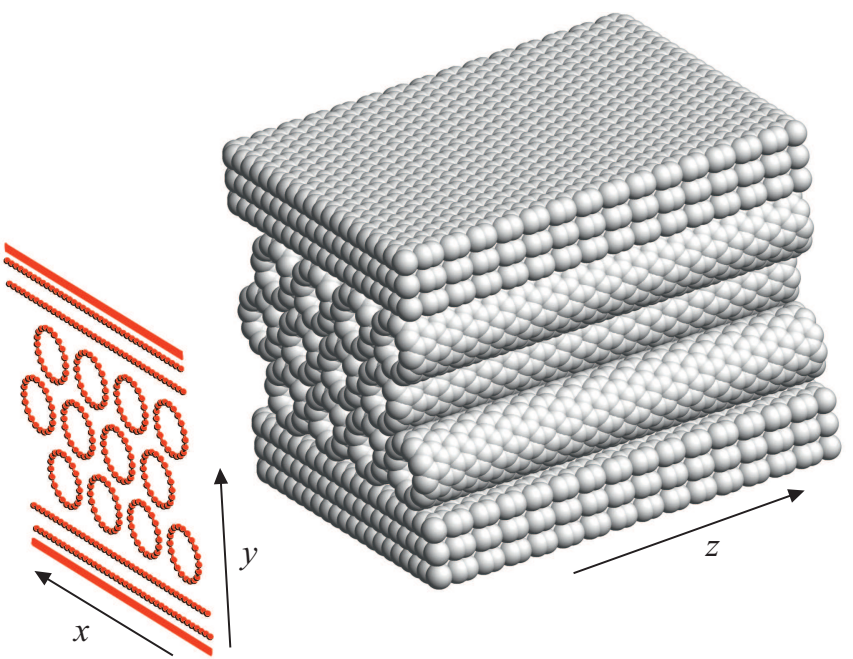

Рис. 1. Схема построения $2 \mathrm{D}$ модели $N_{y}$-слойного массива параллельных УНТ, лежащего между плоскими подложками, образованными многослойными листами графена. Показаны $N_{y}=3$ слоя нанотрубок с индексом хиральности $(10,0)$, лежащие между плоскими трехслойными листами графена, и соответствующая 2D модель системы. Сжатие системы УНТ проводится по оси $y$, по оси $x$ используются периодические граничные условия.

$\left(m_{p}=1.6603 \cdot 10^{-27} \mathrm{~kg}\right.$ - масса протона), число атомов цепи $N_{r}$ определяется из длины наноленты $L=N_{r} R_{0}$.

Потенциал

$$
V(R)=\frac{1}{2} K\left(R-R_{0}\right)^{2}
$$

описывает продольную жесткость цепи, $K$ - жесткость взаимодействия, $R_{0}$ - равновесная длина связи (шаг цепи), $R_{n}=\left|\mathbf{v}_{n}\right|$ - расстояние между соседними узлами $n$ и $n+1$ (вектор $\left.\mathbf{v}_{n}=\mathbf{u}_{n+1}-\mathbf{u}_{n}\right)$.

Потенциал

$$
U(\theta)=\varepsilon_{\theta}[1+\cos (\theta)]
$$

описывает изгибную жесткость цепи, $\theta-$ угол между двумя соседними связями, косинус $n$-го „валентного“ угла $\cos \left(\theta_{n}\right)=-\left(\mathbf{v}_{n-1}, \mathbf{v}_{n}\right) / R_{n-1} R_{n}$.

Параметры потенциалов (2) и (3) определены в [36,41] из анализа дисперсионных кривых наноленты графена: продольная жесткость $K=405 \mathrm{~N} / \mathrm{m}$, энергия $\varepsilon_{\theta}=3.5 \mathrm{eV}$.

В гамильтониане (1) потенциал $W_{0}(y)$ описывает взаимодействие узлов цепи с подложкой, образуемой плоской поверхностью молекулярного кристалла. Будем считать, что поверхность подложки совпадает с плоскостью $y=0$. Для определения этого потенциала была численно найдена зависимость энергии взаимодействия атома углерода с подложкой от его расстояния $y$ до плоской поверхности кристалла. Вычисления [39,42] показали, что энергия взаимодействия $W_{0}(y)$ с хорошей точностью может быть описана потенциалом Леннарда- 
Джонса $(k, l)$

$$
W_{0}(y)=\varepsilon_{0}\left[k\left(d_{0} / y\right)^{l}-l\left(d_{0} / y\right)^{k}\right] /(l-k),
$$

где степень $l>k, \varepsilon_{0}-$ энергия связи атома с подложкой, $d_{0}-$ равновесное расстояние до плоскости подложки. Для плоской поверхности кристалла гексагонального нитрида бора (h-BN) энергия связи $\varepsilon_{0}=0.0903 \mathrm{eV}$, равновесное расстояние $d_{0}=3.45 \AA$, степени $l=10$ и $k=3.75$.

Поперечное сечение УНТ с индексом хиральности $(m, 0)$ состоит из $N_{t}=2 m$ атомов углерода, каждый из которых описывает смещения прямой линии атомов нанотрубки, параллельной оси $z$. Гамильтониан поперечного сечения УНТ (циклической цепи из $N_{t}$ атомов) будет иметь вид

$$
\begin{aligned}
H_{t}= & \sum_{n=1}^{N_{t}}\left[\frac{1}{2} M\left(\dot{\mathbf{u}}_{n}, \dot{\mathbf{u}}_{n}\right)+V\left(R_{n}\right)+U\left(\theta_{n}\right)\right. \\
& \left.+\frac{1}{2} \sum_{l=1,|l-n|>4}^{N_{t}} W_{1}\left(r_{n, l}\right)\right],
\end{aligned}
$$

где потенциал $W_{1}\left(r_{n, l}\right)$ описывает слабые невалентные взаимодействия удаленных узлов цепи $n$ и $l$, $r_{n, l}=\left|u_{l}-u_{n}\right|-$ расстояние между узлами (разница индексов $|n-l|$ определяется с учетом цикличности цепи). Этот потенциал также будет использован для описания взаимодействия между узлами разных цепей. Энергия невалентного взаимодействия узлов цепей с высокой точностью [42] может быть описана потенциалом Леннарда-Джонса $(5,11)$

$$
W_{1}(r)=\varepsilon_{1}\left[5\left(r_{0} / r\right)^{11}-11\left(r_{0} / r\right)^{5}\right] / 6,
$$

с равновесной длиной $r_{0}=3.607 \AA$ и энергией взаимодействия $\varepsilon_{1}=0.00832 \mathrm{eV}$.

\section{3. Модель ограниченного многослойного массива нанотрубок}

Рассмотрим систему параллельных $N_{x y}=N_{x} N_{y}$ одностенных УНТ $(m, 0)$, ограниченную сверху и снизу двухслойными листами графена, расположенную между двумя плоскими подложками $\left(N_{x}-\right.$ число нанотрубок в одном слое, параллельном подложке, $N_{y}$ - число слоев), см. левую часть рис. 1 . По оси $x$ будем использовать периодические граничные условия с периодом $a_{x}$.

Пусть координаты $k$-й наноленты ( $k$-й линейной цепи, $k=1, \ldots, 4)$ задаются $2 N_{r}$-мерным вектором $\mathbf{x}_{k}=\left\{\left(x_{k, n}, y_{k, n}\right)\right\}_{n=1}^{N_{r}}, N_{r} R_{0}=a_{x}$. Тогда энергия деформации наноленты

$$
P_{1}\left(\mathbf{x}_{k}\right)=\sum_{n=1}^{N_{r}}\left[V\left(R_{k, n}\right)+U\left(\theta_{k, n}\right)+W\left(y_{k, n}\right)\right],
$$

где энергия взаимодействия узлов цепи с плоской подложкой $W\left(y_{k, n}\right)=W_{0}\left(y_{k, n}\right)$ для $k=1,2 \quad$ и $W\left(y_{k, n}\right)=W_{0}\left(h-y_{k, n}\right)$ для $k=3,4$. Здесь $h-$ расстояние между поверхностями плоских подложек, первые две цепочки взаимодействуют с первой (низшей) подложкой, вторые две - со второй (верхней) подложкой.

Координаты $k$-й циклической цепи, $(k-4)$-й нанотрубки, задаются $2 N_{t}$-мерным вектором $\mathbf{x}_{k}=\left\{\left(x_{k, n}, y_{k, n}\right)\right\}_{n=1}^{N_{t}}, \quad N_{t}=2 m, \quad k=5,6, \ldots, N_{c}, \quad$ где $N_{c}=4+N_{x y}-$ число всех цепочек. Энергия деформации нанотрубки

$$
P_{2}\left(\mathbf{x}_{k}\right)=\sum_{n=1}^{N_{t}}\left[V\left(R_{k, n}\right)+U\left(\theta_{k, n}\right)+\frac{1}{2} \sum_{l=1,|l-n|>4}^{N_{t}} W_{1}\left(r_{k, n, l}\right)\right] .
$$

Энергия взаимодействия цепочек (нанолент и нанотрубок) с координатами $\mathbf{x}_{k_{i}}=\left\{u_{k_{i}, n}\right\}_{n=1}^{N_{k_{i}}}, i=1,2$,

$$
P_{3}\left(\mathbf{x}_{k_{1}}, \mathbf{x}_{k_{2}}\right)=\sum_{n_{1}=1}^{N_{k_{1}}} \sum_{n_{2}=1}^{N_{k_{2}}} W\left(r_{k_{1}, n_{1} ; k_{2}, n_{2}}\right),
$$

где расстояние между узлами цепей $r_{k_{1}, n_{1} ; k_{2}, n_{2}}=$ $=\left|\mathbf{u}_{k_{2}, n_{2}}-\mathbf{u}_{k_{1}, n_{1}}\right|$, число звеньев в цепи $N_{k}=N_{r}$ при $k=1, \ldots, 4$ и $N_{k}=N_{t}$ при $k=5, \ldots, N_{c}$.

Потенциальная энергия массива нанотрубок с учетом периодического граничного условия по оси $x$ будет иметь вид

$$
\begin{aligned}
E= & \sum_{k=1}^{4} P_{1}\left(\mathbf{x}_{k}\right)+\sum_{k=5}^{N_{c}} P_{2}\left(\mathbf{x}_{k}\right)+P_{3}\left(\mathbf{x}_{1}, \mathbf{x}_{2}\right)+P_{3}\left(\mathbf{x}_{3}, \mathbf{x}_{4}\right) \\
& +\sum_{k_{1}=1}^{4} \sum_{k_{2}=5}^{N_{c}}\left[P_{3}\left(\mathbf{x}_{k_{1}}, \mathbf{x}_{k_{2}}\right)+P_{3}\left(\mathbf{x}_{k_{1}}, \mathbf{x}_{k_{2}}+a_{x} \mathbf{e}_{x}\right)\right] \\
& +\sum_{k_{1}=5}^{N_{c}-1} \sum_{k_{2}=k_{1}+1}^{N_{c}}\left[P_{3}\left(\mathbf{x}_{k_{1}}, \mathbf{x}_{k_{2}}\right)+P_{3}\left(\mathbf{x}_{k_{1}}, \mathbf{x}_{k_{2}}+a_{x} \mathbf{e}_{x}\right)\right],
\end{aligned}
$$

где $2 N_{t}$-мерный вектор $\mathbf{e}_{x}=\{(1,0)\}_{n=1}^{N_{t}}$.

\section{4. Стационарные состояния сжатого многослойного массива нанотрубок}

Для нахождения стационарного состояния многослойного массива УНТ нужно решить задачу на минимум энергии системы

$$
E \rightarrow \min :\left\{\mathbf{x}_{k}\right\}_{k=1}^{N_{c}} .
$$

Задача на минимум энергии (11) решалась численно методом сопряженного градиента. Каждое стационарное состояние массива

$$
\left\{\left[\left(x_{n, k}^{0}, y_{n, k}^{0}\right)\right]_{n=1}^{N_{k}}\right\}_{k=1}^{N_{c}}
$$


будет характеризоваться удельной энергией $E_{a}=E / N_{a}$, где $N_{a}=\sum_{k=1}^{N_{c}} N_{k}=4 N_{r}+N_{x y} N_{t}$ - общее число атомов в системе цепочек, и давлением на плоскости подложки

$$
P=\frac{1}{2 a_{x} a_{z}} \sum_{k=1}^{2} \sum_{n=1}^{N_{r}}\left[W_{0}^{\prime}\left(h-y_{k+2, n}^{0}\right)-W_{0}^{\prime}\left(y_{k, n}^{0}\right)\right],
$$

где штрих обозначает производную функции, $a_{z}=3 r_{c} / 2$ - ширина поперечного сечения массива УНТ.

Анализ возможных стационарных состояний однослойных УНТ на плоской подложке [39] показывает, что нанотрубка с индексом хиральности $(m, 0)$ имеет только одну устойчивую конфигурацию при $m<32$ и две устойчивые (конфигурацию с внутренней полостью и конфигурацию без полости) при $m \geq 32$. Поэтому следует ожидать, что упаковка нанотрубок при $m>32$ (при диаметре $D>2.5 \mathrm{~nm}$ ) может иметь много устойчивых стационарных состояний, которые будут отличаться друг от друга долей нанотрубок, находящихся в коллапсированном состоянии.

Рассмотрим массивы нанотрубок с тремя характерными значениями индекса хиральности $m=15,30,60$ (изолированные нанотрубки с этими индексами имеют диаметры $D=1.17,2.35,4.69 \mathrm{~nm}$ ).

Вначале рассмотрим массив УНТ $(15,0)$ с числом слоев $N_{y}=11$, числом нанотрубок в одном слое $N_{x}=18$ и периодом $a_{x}=26.765 \mathrm{~nm}$ (число атомов в линейной цепи $\left.N_{r}=217\right)-$ см. рис. 2, $a$. Численное решение задачи на минимум энергии (11) показало, что 11-слойный ограниченный массив УНТ без сжатия имеет толщину (расстояние между сжимающими плоскостями) $h_{0}=16 \mathrm{~nm}$. Последовательно уменьшая расстояние $h$ при решении задачи на минимум энергии, мы получим стационарные состояния массива УНТ с любым значением $h<h_{0}$.

Характерный вид стационарного состояния массива УНТ при различных значениях $h$ показан на рис. 2 . Зависимость энергии массива $E_{a}$ и давления на стенки подложки $P$ от $h$ показаны на рис. $3, a, b$. Анализ изменения структуры поперечного сечения массива при уменьшении его толщины показал, что существует два характерных значения $h_{1}=14.9, h_{2}=11.3 \mathrm{~nm}$, при которых меняется тип сжатия массива нанотрубок. При $h>h_{1}$ происходит равномерное сжатие всех нанотрубок, при котором они сохраняют цилиндрическую форму (см. рис. 2,a). При $h_{1}<h<h_{2}$ нанотрубки сжимаются неоднородно, сначала образуются области сильно и слабо сжатых нанотрубок (см. рис. 2, $b$ ), а дальнейшее сжатие приводит к увеличению первой области (см. рис. 2,c). При $h<h_{2}$ область слабо сжатых исчезает, а сильно сжатые нанотрубки сплющиваются - см. рис. 2, d,e. При $h_{2}<h<h_{1}$ энергия массива $E_{a}$ линейно растет с уменьшением толщины, а давление на стенки $P$ остается почти постоянным.

Аналогичный вид также имеют зависимости энергии $E_{a}$ и давления $P$ от толщины 11-слойного массива
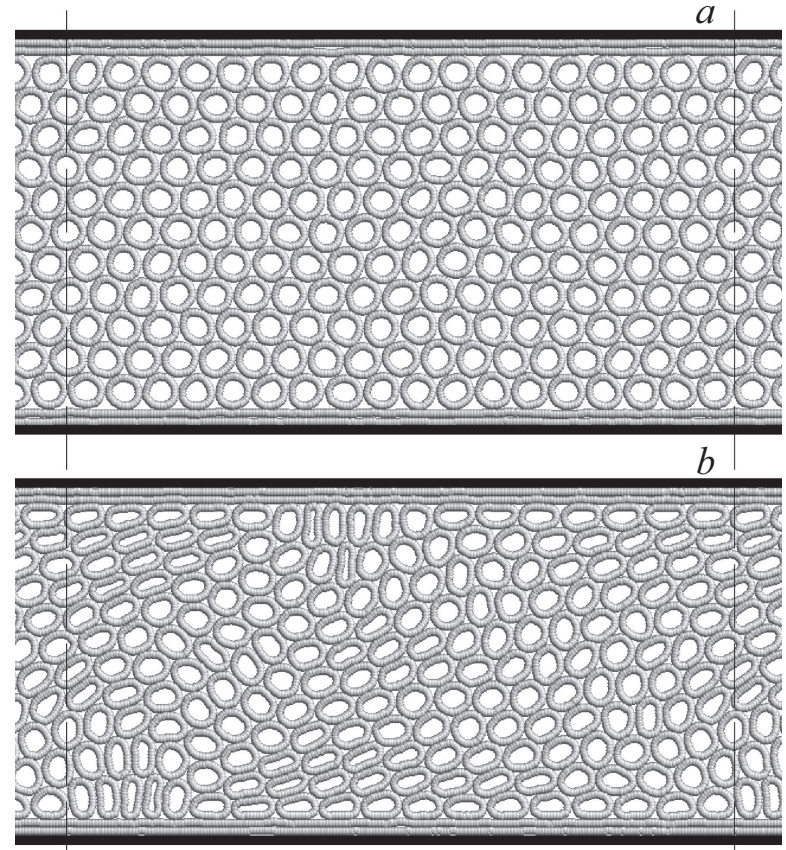

$c$

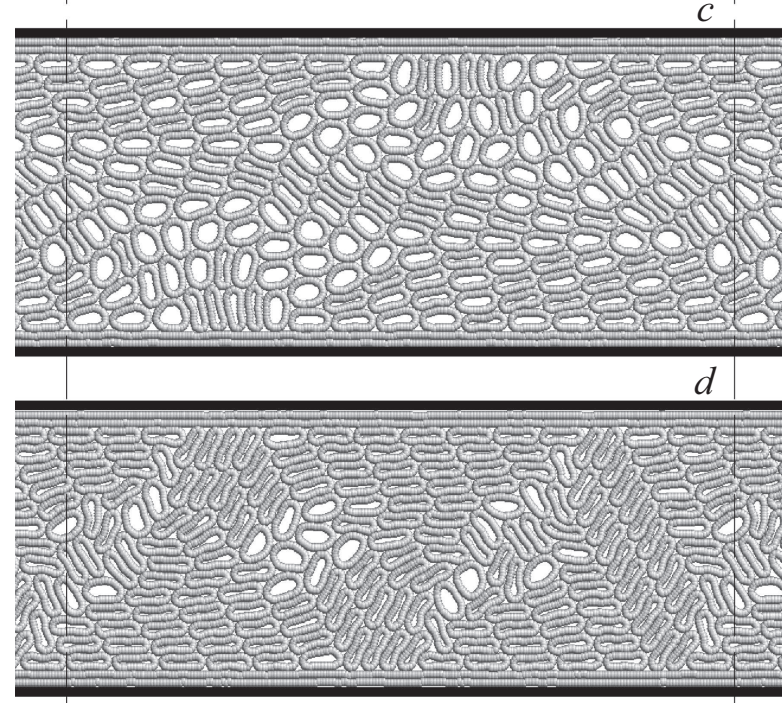

$e$

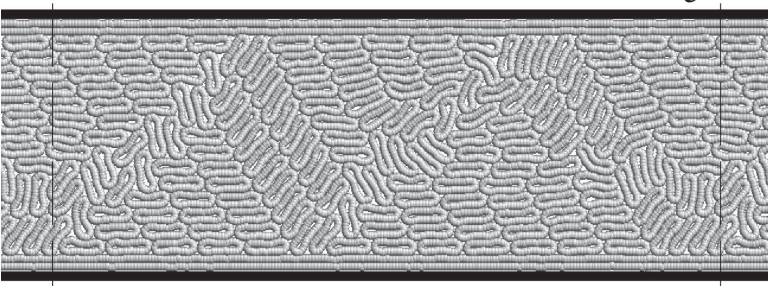

Рис. 2. Вид стационарных состояний слоистой структуры из $N_{x} \times N_{y}$ нанотрубок с индексом хиральности $(15,0)$ и двух граничных двухслойных листов графена (число нанотрубок в одном слое $N_{x}=18$, число слоев $N_{y}=11$, число звеньев в каждой циклической цепи $N_{t}=30$, число звеньев в линейной цепи $\left.N_{r}=217\right)$ при расстоянии между сжимающими плоскостями подложек: $(a)-h=15.87,(b)-14.27,(c)-12.77,(d)-$ 11.37 и $(e)-10.37 \mathrm{~nm}$. Толстые горизонтальные прямые показывают сжимающие плоскости, пунктирные вертикальные границы периодической ячейки (период $a_{x}=26.765 \mathrm{~nm}$ ) 
нанотрубок $(30,0)-$ см. рис. 4, $a, b$. Здесь характерные значения толщины $h_{0}=26.6, h_{1}=25.8$ и $h_{2}=15.0 \mathrm{~nm}$. Отметим, что сжатие массива нанотрубок $(15,0)$ и $(30,0)$ происходит упруго - при освобождении сжимающих стенок массив всегда возвращается в основное несжатое состояние.

Сжатие массива нанотрубок большого диаметра происходит по другому сценарию, что связано с их бистабильностью (нанотрубки могут находиться в двух

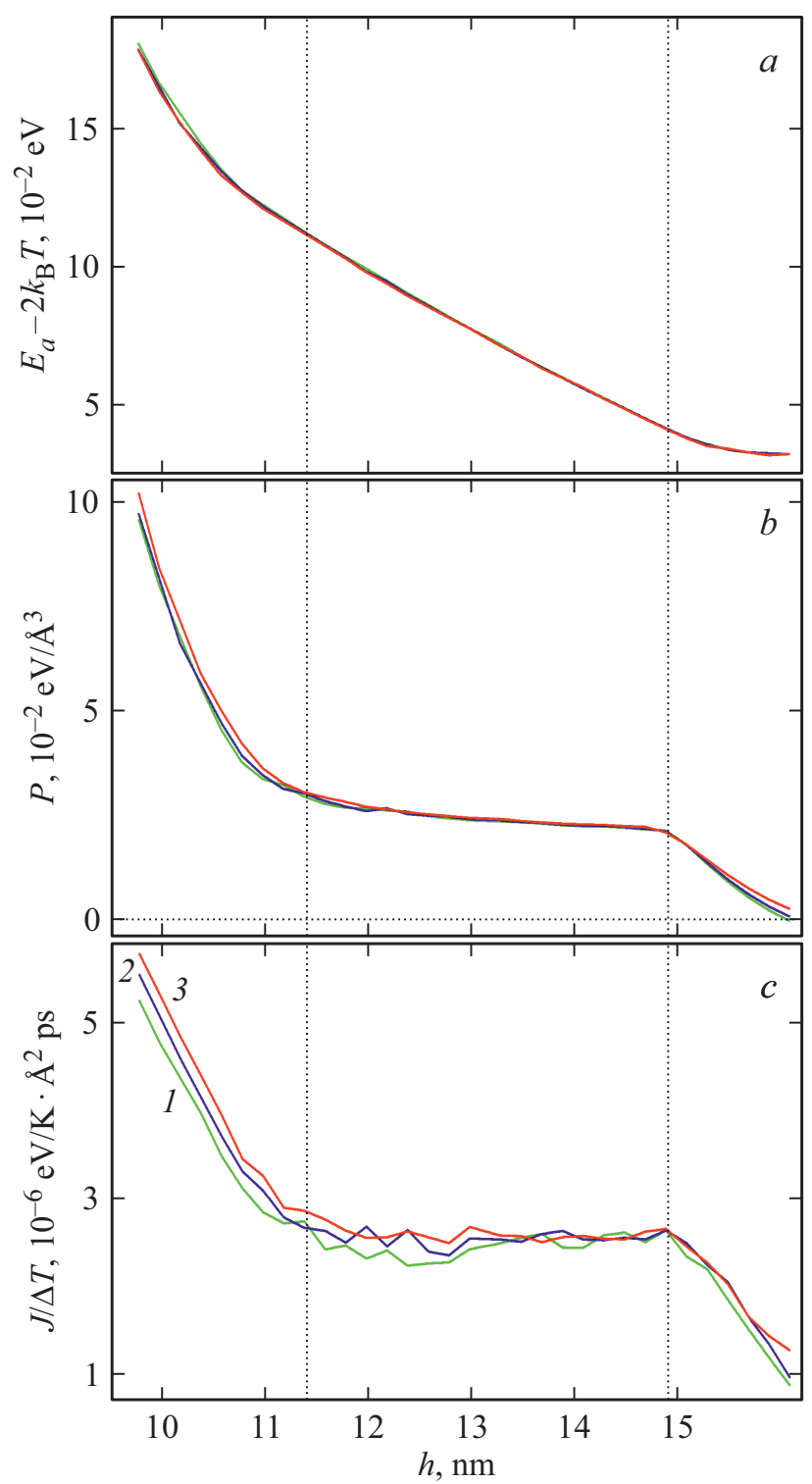

Рис. 3. Зависимость $(a)$ удельной энергии $E_{a},(b)$ давления $P$ и $(c)$ величины теплового потока $J$ для слоистой структуры $N_{x} \times N_{y}$ нанотрубок $(15,0)$ от расстояния между сжимающими плоскостями $h$ при температуре граничных термостатов $T_{ \pm}=T \pm \Delta T\left(N_{x}=18, N_{y}=11, N_{t}=30\right.$, $\left.N_{r}=217, a_{x}=26.765 \mathrm{~nm}\right)$. Кривая 1 дает зависимость при $T_{ \pm}=150 \pm 15 \mathrm{~K}$, кривая $2-$ при $T_{ \pm}=300 \pm 30 \mathrm{~K}$, кривая $3-$ при $T_{ \pm}=600 \pm 60 \mathrm{~K}$. Вертикальные пунктирные линии показывают характерные значения $h=h_{1}=14.9$ и $h=h_{2}=11.4 \mathrm{~nm}$.

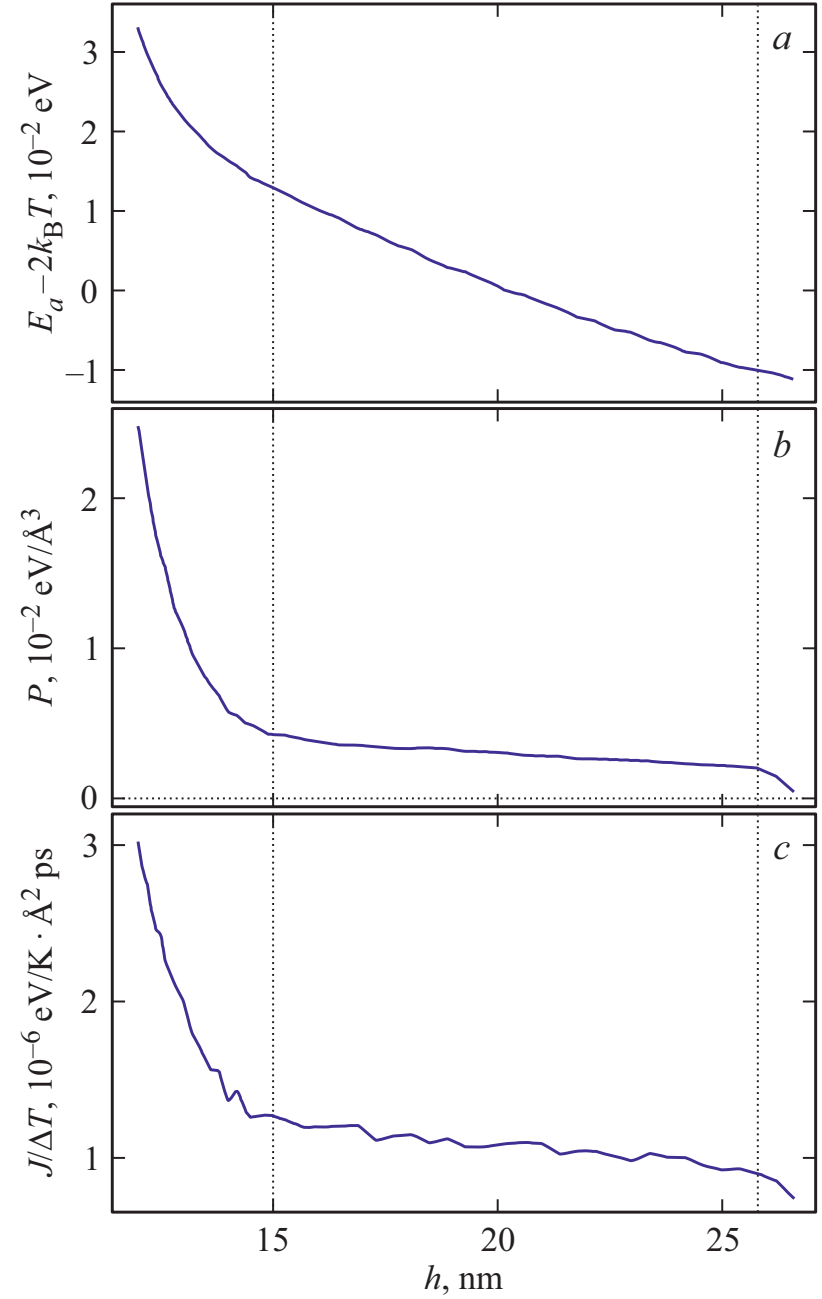

Рис. 4. Зависимость $(a)$ удельной энергии $E_{a},(b)$ давления $P$ и $(c)$ величины теплового потока $J$ для слоистой структуры $N_{x} \times N_{y}$ нанотрубок $(30,0)$ от расстояния между сжимающими плоскостями $h$ при температуре граничных термостатов $T_{ \pm}=300 \pm 30 \mathrm{~K}\left(N_{x}=18, N_{y}=11, N_{t}=60, N_{r}=392\right.$, $\left.a_{x}=48.145 \mathrm{~nm}\right)$. Вертикальные пунктирные линии показывают характерные значения $h=h_{1}=25.8$ и $h=h_{2}=15.0 \mathrm{~nm}$.

устойчивых состояниях: в открытом (цилиндрическом) и в коллапсированном (схлопнутом) состояниях). Сжатие массива УНТ $(60,0)$ происходит за счет коллапсирования части нанотрубок. Число коллапсированных нанотрубок монотонно возрастает с уменьшением $h$, а энергия массива вначале незначительно убывает, затем также возрастает, а давление на сжимающие стенки при этом не меняется - cм. рис. 5, $a, b$. Так происходит до достижения характерного значения толщины массива $h=h_{2}$, при котором все нанотрубки уже оказываются в коллапсированном состоянии. Дальнейшее сжатие уже приводит к резкому росту энергии и давления.

Массив из $N_{y}=11$ слоев открытых нанотрубок $(60,0)$ имеет толщину $h_{0}=38.9 \mathrm{~nm}$. При $h>h_{2}=16 \mathrm{~nm}$ сжатие массива происходит только за счет роста доли коллапсированных нанотрубок. Такое сжатие не является 


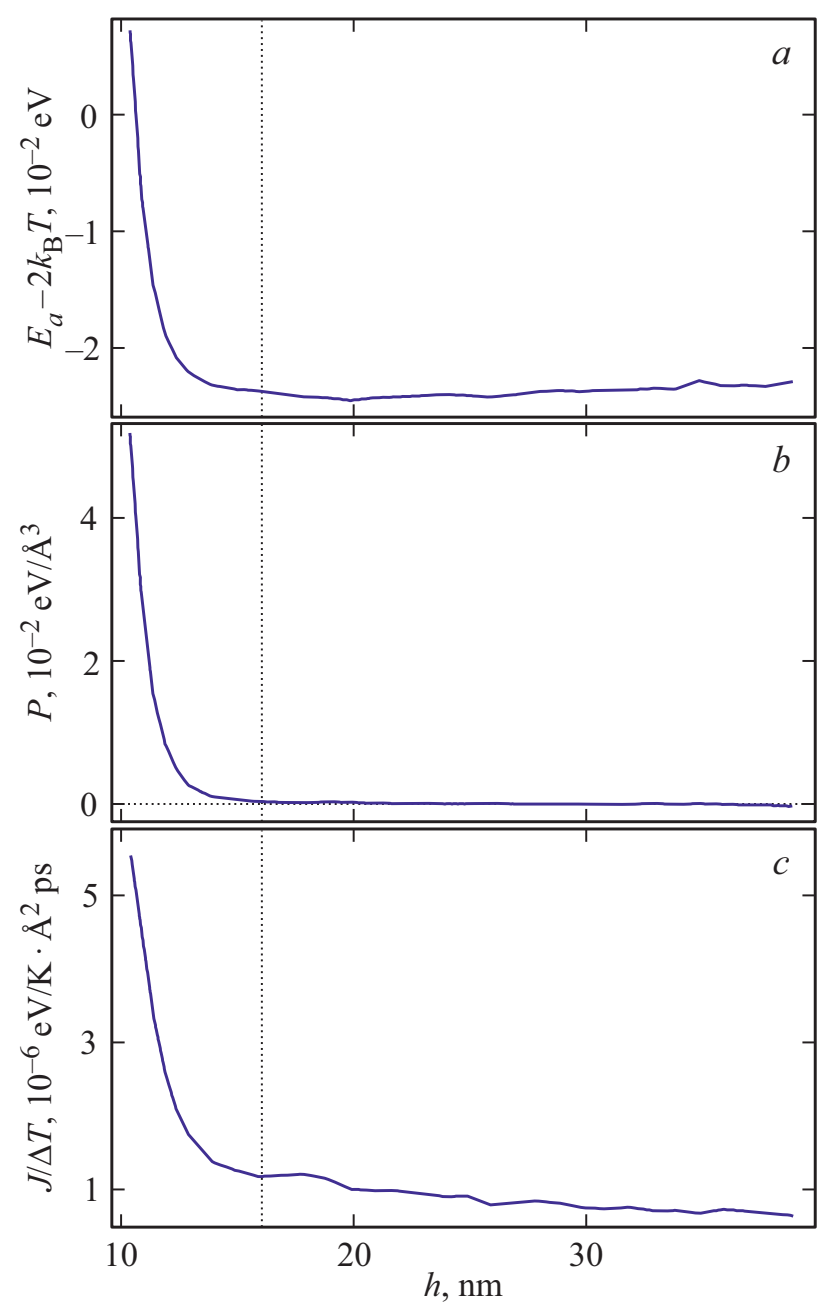

Рис. 5. Зависимость $(a)$ удельной энергии $E_{a},(b)$ давления $P$ и $(c)$ величины теплового потока $J$ для слоистой структуры $N_{x} \times N_{y}$ нанотрубок $(60,0)$ от расстояния между сжимающими плоскостями $h$ при температуре граничных термостатов $T_{ \pm}=300 \pm 30 \mathrm{~K} \quad\left(N_{x}=18, N_{y}=11, N_{t}=120\right.$, $\left.N_{r}=841, a_{x}=103.294 \mathrm{~nm}\right)$. Вертикальные пунктирные линии показывают значение $h=h_{2}=16.0 \mathrm{~nm}$.

упругим, оно только за счет коллапса части нанотрубок переводит массив из одного устойчивого состояния в другое. Сжатие становится упругим только при $h<h_{2}$, когда все нанотрубки в массиве оказываются в коллапсированном состоянии.

\section{5. Теплоперенос поперек многослойного массива УНТ}

Промоделируем теплоперенос поперек многослойного массива УНТ и проанализируем зависимость теплового потока от толщины сжатого массива. Для этого самую низшую линейную цепочку с номером $k=1$ поместим в термостат Ланжевена температуры $T_{+}=T+\Delta T$, а самую верхнюю цепочку с $k=4-$ в термостат с тем- пературой $T_{-}=T-\Delta T$, где $T-$ средняя температура системы, а $2 \Delta T$ - разница температуры термостатов.

Динамика рассматриваемой системы молекулярных цепей описывается системой уравнений Ланжевена

$$
\begin{gathered}
M \ddot{\mathbf{x}}_{k}=-\frac{\partial E}{\partial \mathbf{x}_{k}}-\Gamma M \dot{\mathbf{x}}_{k}-\Xi_{k}, \quad k=1,4 \\
M \ddot{\mathbf{x}}_{k}=-\frac{\partial E}{\partial \mathbf{x}_{k}}, \quad k=2,3,5,6, \ldots, N_{c},
\end{gathered}
$$

где $x_{k}-2 N_{k}$-мерный вектор, задающий координаты $k$-той цепи, $E$ - потенциальная энергия системы цепей $(10), \Gamma=1 / t_{r}-$ коэффициент трения, характеризующий интенсивность взаимодействия крайних линейных цепей с термостатами (время релаксации скорости частицы из-за взаимодействия с термостатом $\left.t_{r}=1 \mathrm{ps}\right), \Xi_{k}=\left\{\left(\xi_{k, n, 1}, \xi_{k, n, 2}\right)\right\}_{n=1}^{N_{r}}-2 N_{r}$-мерный вектор нормально распределенных случайных сил Ланжевена с функциями корреляции

$$
\left\langle\xi_{k_{1}, n_{1}, i}\left(t_{1}\right) \xi_{k_{2}, n_{2}, j}\left(t_{2}\right)\right\rangle=2 M k_{B} T_{k_{1}} \Gamma \delta_{k_{1} k_{2}} \delta_{n_{1} n_{2}} \delta_{i j} \delta\left(t_{1}-t_{2}\right)
$$

$\left(k_{B}\right.$ - постоянная Больцмана, $T_{k}=T \pm \Delta T-$ температура термостата).

Величину теплового потока через массив удобно определить как величину теплового потока между краевыми линейными цепочками с $k=1,2$ и $k=3,4$

$$
\begin{aligned}
& J_{1,2}=\sum_{n_{1}=1}^{N_{r}} \sum_{n_{2}=1}^{N_{r}}\left(\dot{\mathbf{u}}_{2, n_{2}}, \mathbf{F}_{1, n_{1} ; 2, n_{2}}\right), \\
& J_{3,4}=\sum_{n_{1}=1}^{N_{r}} \sum_{n_{2}=1}^{N_{r}}\left(\dot{\mathbf{u}}_{4, n_{2}}, \mathbf{F}_{3, n_{1} ; 4, n_{2}}\right),
\end{aligned}
$$

где вектор сил

$$
\mathbf{F}_{k_{1}, n_{1} ; k_{2}, n_{2}}=\left(\mathbf{u}_{k_{2}, n_{2}}-\mathbf{u}_{k_{1}, n_{1}}\right) W_{1}^{\prime}\left(r_{k_{1}, n_{1} ; k_{2}, n_{2}}\right) / r_{k_{1}, n_{1} ; k_{2}, n_{2}},
$$

a $r_{k_{1}, n_{1} ; k_{2}, n_{2}}=\left|\mathbf{u}_{k_{2}, n_{2}}-\mathbf{u}_{k_{1}, n_{1}}\right|-$ расстояние между узлом $n_{1}$ цепи $k_{1}$ и узлом $n_{2}$ цепи $k_{2}$.

Проинтегрируем численно систему уравнений движения (12), (13). В качестве начального условия возьмем стационарное состояние сжатого массива нанотрубок $\left\{\mathbf{x}_{k}^{0}\right\}_{k=1}^{N_{c}}$ (положим $\left.\left\{\mathbf{x}_{k}(0)=\mathbf{x}_{k}^{0}, \dot{\mathbf{x}}_{k}(0)=\mathbf{0}\right\}_{k=1}^{N_{c}}\right)$. Вначале проинтегрируем систему уравнений движения в течение времени $t_{0}$ до образования в массиве линейного температурного профиля и стационарного теплового потока, интегрируя далее, найдем распределение температуры в системе цепей

$$
T_{k}=\lim _{t \rightarrow \infty} \frac{M}{2 N_{k} k_{B}\left(t-t_{0}\right)} \int_{t_{0}}^{t_{0}+t}\left(\dot{\mathbf{x}}_{k}(\tau), \dot{\mathbf{x}}_{k}(\tau)\right) d \tau,
$$

$k=1,2, \ldots, N_{c}$, и величину удельного теплового потока через массив УНТ

$$
J=\left\langle J_{1,2}\right\rangle=\left\langle J_{3,4}\right\rangle
$$



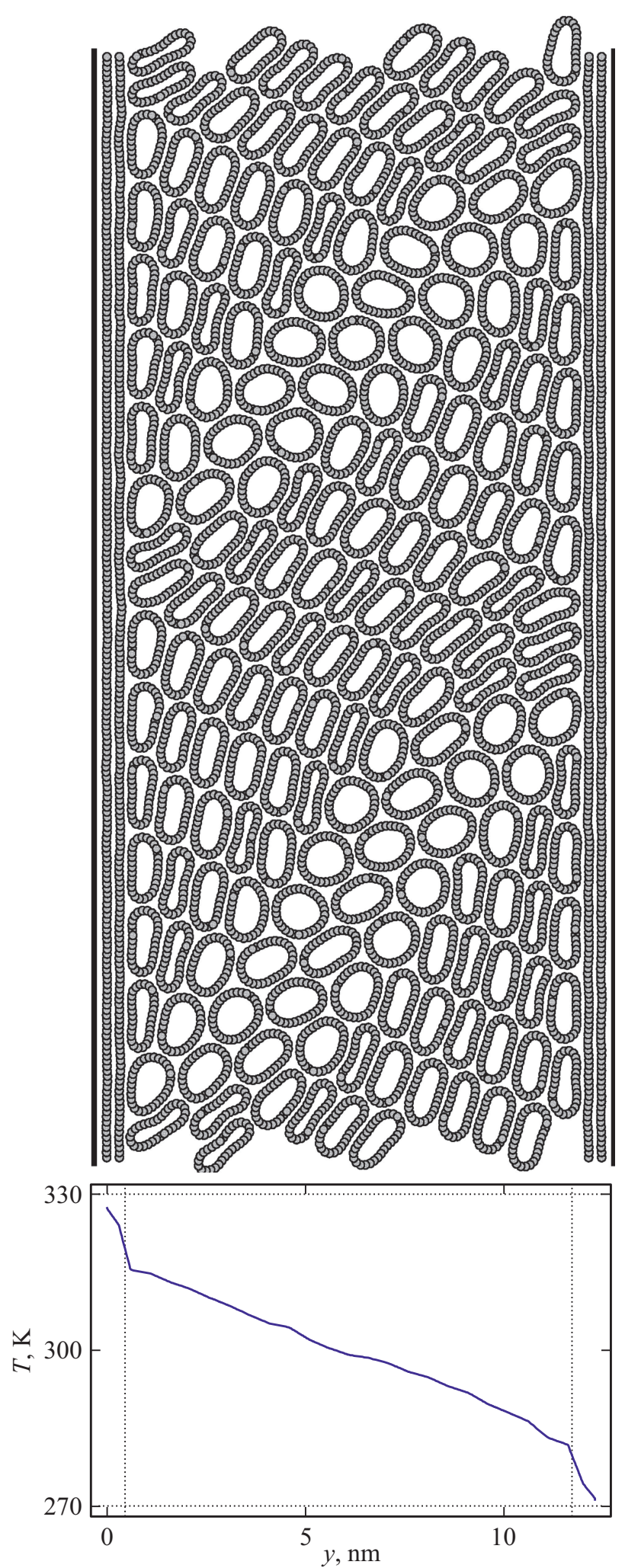

Рис. 6. Поперечный температурный профиль сжатой слоистой структуры из $N_{x} \times N_{y}$ нанотрубок с индексом хиральности $(15,0)$ и двух граничных двухслойных листов графена $\left(N_{x}=18, N_{y}=11, N_{t}=30, N_{r}=217\right)$ при расстоянии между сжимающими плоскостями $h=12.97 \mathrm{~nm}$. Вверху показан один период слоистой структуры $\left(a_{x}=26.765 \mathrm{~nm}\right)$, внизу - зависимость температуры структуры $T$ от поперечной координаты $y$. Температура краевых термостатов $T_{ \pm}=300 \pm 30 \mathrm{~K}$. где величина теплового потока между соседними линейными цепями

$$
\left\langle J_{k_{1}, k_{2}}\right\rangle=\lim _{t \rightarrow \infty} \frac{1}{\left(t-t_{0}\right) a_{x} a_{z}} \int_{t_{0}}^{t_{0}+t} J_{k_{1}, k_{2}}(\tau) d \tau .
$$

Выполнение равенства (14) может служить критерием точности вычислений.

Характерное распределение температуры поперек многослойного массива УНТ показано на рис. 6. Как видно на рисунке, в массиве между ограничивающими его двухслойными листами графена образуется линейный градиент температуры.

Численное моделирование теплопереноса показало, что вид полученных ранее зависимостей удельной энергии $E_{a}$ и давления на сжимающие плоскости $P$ от расстояния между ними (от толщины массива УНТ) $h$ практически не зависит от значения температуры - см. pис. $3, a, b$. Величина теплового потока поперек массива, нормированная на разницу температуры краевых термостатов $J / \Delta T$, тоже слабо зависит от среднего значения температуры массива $T$, но существенно зависит от величины поперечного сжатия массива $h-$ см. рис. $3, c$.

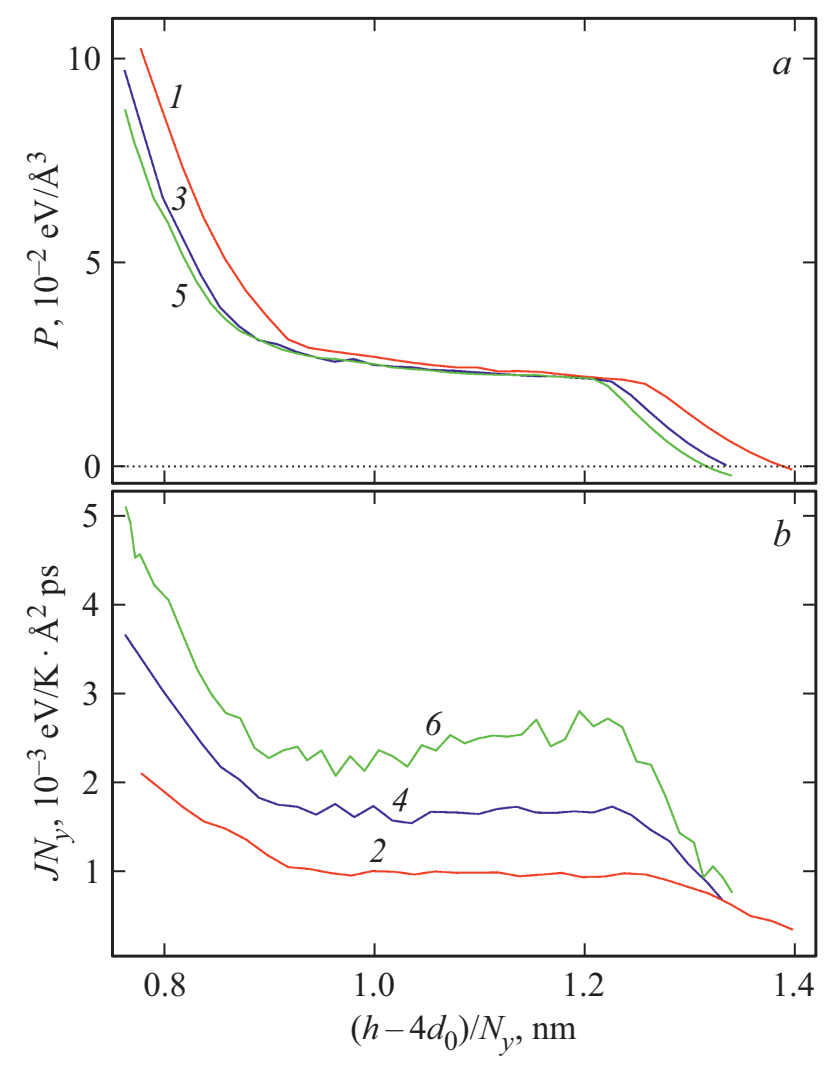

Рис. 7. Зависимость $(a)$ давления $P$ и $(b)$ кратной числу слоев величины теплового потока $J N_{y}$ для слоистой структуры $N_{x} \times N_{y}$ нанотрубок $(15,0)$ от относительного сжатия массива $\left(h-4 d_{0}\right) / N_{y}$ при температуре граничных термостатов $T_{ \pm}=300 \pm 30 \mathrm{~K}\left(N_{x}=18, N_{t}=30, N_{r}=217, a_{x}=26.8 \mathrm{~nm}\right)$. Кривые 1 и 2 для структуры из $N_{y}=5$ слоев, кривые 3 и $4-$ для $N_{y}=11$, кривые 5 и 6 - для $N_{y}=22$. 
В массиве нанотрубок с индексом хиральности $(15,0)$ (в массиве нанотрубок малого диаметра) при $h>h_{1}$ и $h<h_{2}$, когда происходит однородное сжатие всех нанотрубок, величина теплового потока $J$ линейно растет при сближении сжимающих массив стенок (при уменьшении $h$ ). Но при $h_{2}<h<h_{1}$, когда происходит неоднородное сжатие массива УНТ, величина теплового потока практически не меняется при сближении стенок - см. рис. 3,c. При увеличении числа слоев нанотрубок массива величина теплового потока может даже уменьшаться при поперечном сжатии массива см. рис. $7, b$. Отметим, что зависимость давления $P$ на сжимающие стенки от относительного поперечного сжатия массива $\left(h-4 d_{0}\right) / N_{y}$ практически не зависит от числа слоев $N_{y}-$ см. рис. 7, $a$. Из рисунка также следует, что при всех значениях относительного сжатия величина теплового потока, умноженная на число слоев $J N_{y}$ возрастает с увеличением числа слоев, т.е. при рассмотренном максимальном числе слоев $N_{y}=22$ сходимости поперечной теплопроводности массива УНТ не происходит (при сходимости должен существовать конечный предел $\left.\lim _{N_{y} \rightarrow \infty} J N_{y}\right)$.

Для нанотрубок $(30,0)$ и $(60,0)$ поперечное сжатие их многослойных массивов при $h>h_{2}$ всегда приводит к медленному, но линейному росту величины теплового потока $J$ - см. рис. 4, c и 5, c. Поэтому наиболее эффективно изменять величину теплового потока при поперечном сжатии можно только для многослойных массивов нанотрубок небольшого диаметра (для УНТ $(15,0)$ ).

\section{6. Заключение}

Проведенное исследование показывает, что поперечное сжатие многослойного массива УНТ может происходить однородно, когда все нанотрубки в массиве одинаково сжимаются, и неоднородно, когда часть нанотрубок сжимаются сильно, а часть - слабо. Однородный сценарий сжатия реализуется при слабом, когда все нанотрубки остаются в открытом состоянии, или при очень сильном сжатии, когда все нанотрубки находятся в сплющенном состоянии. При среднем сжатии всегда реализуется неоднородный сценарий. Численное моделирование теплопереноса поперек массива показало, что при однородном сжатии массива его теплопроводность увеличивается. При неоднородном сжатии массива теплопроводность не меняется, а при большом числе слоев нанотрубок может даже уменьшаться. Этот эффект особенно ярко проявляется для массивов нанотрубок небольшого диаметра $(D<2 \mathrm{~nm})$.

\section{Финансирование работы}

Работа выполнена при финансовой поддержке Российского научного фонда (проект 18-29-19135). Вычислительные ресурсы предоставлены межведомственным суперкомпьютерным центром РАН.

\section{Конфликт интересов}

Автор заявляет об отсутствии конфликта интересов.

\section{Список литературы}

[1] Л.В. Радушкевич, В.М. Лукьянович. ЖФХ 26, 1, 88-95 (1952).

[2] S. Iijima. Nature 354, 56 (1991).

[3] А.В. Елецкий. УФН 172, 4, 401 (2002).

[4] D. Qian, G.J. Wagner, W.K. Liu, M.-F. Yu, R.S. Ruoff. Appl. Mech. Rev. 55, 495 (2002).

[5] J. Di, S. Fang, F.A. Moura, D.S. Galvao, J. Bykova, A. Aliev, M.J.d. Andrade, X. Lepro, N. Li, C. Haines, R. Ovalle-Robles, D. Qian, R.H. Baughman. Adv. Mater. 28, 6598 (2016).

[6] Y. Bai, R. Zhang, X. Ye, Z. Zhu, H. Xie, B. Shen, D. Cai, B. Liu, C. Zhang, Z. Jia, S. Zhang, X. Li, F. Wei. Nature Nanotechnology 13, 589 (2018).

[7] B.C. Liu, T.J. Lee, S.H. Lee, C.Y. Park, C.J. Lee. Chem. Phys. Lett. 377, 55 (2003).

[8] Y. Li, X. Zhang, X. Tao, J. Xu, W. Huang, J. Luo, Z. Luo, T. Li, F. Liu, Y. Bao, H.J. Geise. Carbon 43, 2, 295 (2005).

[9] Э.Г. Раков. Успехи химии 82, 1, 27 (2013).

[10] C. Yu, L. Shi, Z. Yao, D. Li, A. Majumdar. Nano Lett. 5, 9, 1842 (2005).

[11] E. Pop, D. Mann, Q. Wang, K. Goodson, H. Dai. Nano Lett. 6, 1, 96 (2006).

[12] Q. Li, C. Liu, X. Wang, S. Fan. Nanotechnology 20, 14, 145702 (2009).

[13] M.T. Pettes, L. Shi. Adv. Funct. Mater. 19, 24, 3918 (2009).

[14] B. Kumanek, D. Janas. J. Mater. Sci. 54, 107397 (2019).

[15] G. Zhang, B. Li. J. Chem. Phys. 123, 114714 (2005).

[16] J.R. Lukes, H. Zhong. J. Heat Transfer. 129, 6, 705 (2007).

[17] A.V. Savin, B. Hu, Y.S. Kivshar. Phys. Rev. B, 80, 195423 (2009).

[18] А.В. Савин, О.И. Савина. ФТТ 61, 2, 409 (2019).

[19] S. Badaire, V. Pichot, C. Zakri, P. Poulin, P. Launois, J. Vavro, C. Guthy, M. Chen, J.E. Fischer. J. Appl. Phys. 96, 12, 7509 (2004).

[20] A.E. Aliev, C. Guthy, M. Zhang, S. Fang, A.A. Zakhidov, J.E. Fischer, R.H. Baughman. Carbon 45, 15, 2880 (2007).

[21] A. Duzynska, A. Taube, K.P. Korona, J. Judek, M. Zdrojek. Appl. Phys. Lett. 106, 18, 183108 (2015).

[22] F. Lian, J.P. Llinas, Z. Li, D. Estrada, E. Pop. Appl. Phys. Lett. 108, 10, 103101 (2016).

[23] H. Zhan, Y.W. Chen, Q.Q. Shi, Y. Zhang, R.W. Mo, J.N. Wang. Carbon 186, 205 (2022).

[24] W.J. Evans, M. Shen, P. Keblinski. Appl. Phys. Lett. 100, 261908 (2012).

[25] M.R. Gharib-Zahedi, M. Tafazzoli, M.C. Bohm, M. Alaghemandi. J. Chem. Phys. 139, 184704 (2013).

[26] J. Wang, D. Chen, J. Wallace, J. Gigax, X. Wang, L. Shao. Appl. Phys. Lett. 104, 191902 (2014).

[27] N.S. Chopra, L.X. Benedict, V.H. Crespi, M.L. Cohen, S.G. Louie, A. Zettl. Nature 377, 14, 135 (1995).

[28] G. Gao, T. Çağin, W.A. Goddard III. Nanotechnology 9, 184 (1998).

[29] J. Xiao, B. Liu, Y. Huang, J. Zuo, K.-C. Hwang, M.-F. Yu. Nanotechnology 18, 395703 (2007).

[30] T. Chang. Phys. Rev. Lett. 101, 175501 (2008). 
[31] J.A. Baimova, Q. Fan,1 L. Zeng, Z. Wang, S.V. Dmitriev, X. Feng, K. Zhou. J. Nanomater. 2015, 186231 (2015).

[32] A. Impellizzeri, P. Briddon, C.P. Ewels, Phys. Rev. B 100, 115410 (2019).

[33] M.M. Maslov, K.S. Grishakov, M.A. Gimaldinova, K.P. Katin. Fuller. Nanotub. Car. Nanostructures 28, 97 (2020).

[34] A.Y. Cao, P.L. Dickrell, W.G. Sawyer, M.N. Ghasemi-Nejhad, P.M. Ajayan, Science 310, 1307 (2005).

[35] L.K. Rysaeva, E.A. Korznikova, R.T. Murzaev, D.U. Abdullina, A.A. Kudreyko, J.A. Baimova, D.S. Lisovenko, S.V. Dmitriev. Facta Univ. Ser. Mech. Eng. 18, 1 (2020).

[36] A.V. Savin, E.A. Korznikova, S.V. Dmitriev. Phys. Rev. B 92 , 035412, (2015).

[37] E.A. Korznikova, L.K. Rysaeva, A.V. Savin, E.G. Soboleva, E.G. Ekomasov, M.A. Ilgamov, S.V. Dmitriev. Materials 12, 3951 (2019).

[38] A. Savin, E. Korznikova, S. Dmitriev, E. Soboleva, Comp. Mater. Sci. 135, 99 (2017).

[39] А.В. Савин, О.И. Савина. ФТТ 61, 11, 2257 (2019).

[40] А.В. Савин, О.И. Савина. ФТТ 63, 1, 137 (2021).

[41] А.В. Савин, Е.А. Корзникова, С.В. Дмитриев. ФТТ 57, 11, 2278 (2015).

[42] A.V. Savin, E.A. Korznikova, S.V. Dmitriev. Phys. Rev. B 99, 235411 (2019).

Редактор Т.Н. Василевская 